\title{
Thomas Anz
}

\section{Literarische Norm und Autonomie. Individualitätsspielräume in der modernisierten Literaturgesellschaft des 18 . Jahrhunderts}

Die Literaturgeschichtsschreibung stellt das Verhältnis von literarischer Norm und Autonomie im 18. Jahrhundert in Anlehnung an das damalige Selbstverständnis gewöhnlich so dar: Etwa um 1750 wird das bis dahin dominante Paradigma einer klassizistischen Regelpoetik abgelöst von dem der Genieāsthetik ${ }^{1}$. Die sich überbietende Kritik tradierter Autoritäten radikalisiert sich seit der Jahrhundertmitte zu einer Ästhetik, die das Dichter-„,Genie“ endgültig von allen normativen Bindungen befreit sieht. „Die Darstellung des Weges von anfänglich noch starker Orientierung am tradierten Regelsystem bis zu einer als schöpferisch verstandenen Freiheit von allen Normen ergibt die Geschichte der Literaturästhetik im 18. Jahrhundert. ${ }^{\text {“2 }}$ So heißt es noch 1985 in der monumentalen „Geschichte des Genie-Gedankens“ von Jochen Schmidt.

Gottscheds mit der Autorität frühaufklārerischer Rationalität legitimierter Regelrigorismus repräsentiert bekanntlich die Position, gegenüber der die Genieästhetik ihr Profil gewinnt. Und mit Gottsched zugleich verfällt auch die von ihm (aus anderen Gründen) attackierte Barockliteratur dem genieāsthetischen Verdikt. Ihre enge Bindung an die Disziplinen der Rhetorik vertrug sich nicht mit dem Affekt des Genies gegen Anweisungen, Vorschriften und Rezepte jeglicher Art.

Der Gegensatz von Rhetorik und Regelpoetik auf der einen und Genieästhetik auf der anderen Seite fand in zahllosen Begriffsdichotomien seine Entsprechungen und Konkretisierungen: in den Gegenüberstellungen des „poeta doctus“ und des „poeta

Der Beitrag arbeitet mit zum Teil wörtlichen Übernahmen einige Überlegungen meines Aufsatzes „Vorschläge zur Grundlegung einer Soziologie literarischer Normen“ aus (in: Internationales Archiv für Sozialgeschichte der deutschen Literatur 9 (1984) 128-144; im folgenden zitiert: $A n z$, Soziologie literarischer Normen). Anregungen zur literaturwissenschaftlichen Adaptation sozialwissenschaftlicher Modernisierungstheorien verdankt er der Münchener Forschergruppe "Sozialgeschichte der deutschen Literatur 1770-1900“.

'Von einem „kunsttheoretischen Paradigmawechsel um 1750“ spricht Günter Peters, Der zerrissene Engel. Genieästhetik und literarische Selbstdarstellung im achtzehnten Jahrhundert (Stuttgart 1982) $68 \mathrm{ff}$.

${ }_{2}^{2}$ Jochen Schmidt, Die Geschichte des Genie-Gedankens 1750-1945. Band 1, Von der Aufklārung bis zum Idealismus (Darmstadt 1985) 9; im folgenden zitiert: Schmidt, Geschichte des Genie-Gedankens. 
vates“, des „Hofdichters“ und des „freien Schriftstellers“, der Nachahmung und der Schöpfung, des Wahrscheinlichen und des Wunderbaren, der Rationalität und der Einbildungskraft, des Typischen und des Individuellen, der Vernunft und des Gefühls, der Moralitāt und der Leidenschaft, des Mechanischen und des Organischen, des Alten und des Neuen, der Traditionalität und der Modernität, der Orientierung an vorbildlichen Mustern und der innovativen Originalität und so fort.

Die intersubjektive Verbindlichkeit von Normen und der Anspruch auf literarische Autonomie schließen sich innerhalb derart dichotomischer Denkformen aus. Das autonome Dichterindividuum, dem das Prādikat „Genie“ zuerkannt wird, macht sich frei von allen vorgegebenen Ansprüchen, es schafft sich, unbeeindruckt von potentiellen Sanktionen, seine Regeln selbst. Die Wirkungsgeschichte dieser dichotomischen Denkfiguren reicht, gestārkt durch die romantische Programmatik, bis in die Gegenwart. Friedrich Schlegels oft zitiertes Diktum über die romantische Dichtart, sie allein sei frei und erkenne als ihr erstes Gesetz an, „daß die Willkür des Dichters kein Gesetz über sich leide“ ${ }^{\star 3}$, wird noch heute gerne von Künstlern mit gleichartigen Formulierungen zur Kennzeichnung ihres Selbstverständnisses wiederholt. Doch nicht nur die Geschichte der Literatur, sondern auch die der Geistes- und Sozialwissenschaften zeigt sich von diesen Denkfiguren nachhaltig geprägt. Die Differenz von (niedrig bewerteter) Normgebundenheit und (hoch bewerteter) Normfreiheit kehrt in existentialistischen Unterscheidungen von uneigentlichem Dasein und eigentlicher Existenz ebenso wieder wie noch in jüngsten, aufklärungskritischen und gerne auch „postmodern" genannten Gegenüberstellungen von zivilisierter Vernunft und dem unzivilisierten "Anderen der Vernunft“, das man, angeregt von Foucault, unter anderem mit dem „Wahnsinn“ identifiziert. Das von Foucault inspirierte Mißtrauen gegenüber der Ordnung des vernünftigen Diskurses mit den ihr eingelagerten Macht- und Kontrolltechniken läßt sich auf die genieästhetischen Verdikte gegen die von Gottsched im Namen der ,gesunden' Vernunft postulierten Regeln zwanglos rückprojizieren.

Was die Literaturwissenschaft angeht, so hatte sich $z$ wischen ihr und ihren historischen Gegenständen aus dem 18. Jahrhundert ein zirkulärer Mechanismus gegenseitiger Bestätigungen entwickelt, der nur zögernd aufgebrochen werden konnte. Dem Normbegriff jedenfalls stand sie lange (und steht sie zum Teil noch heute) ähnlich reserviert gegenüber wie die Genieästhetik dem der Regel. Autonomie, Individualität und Innovation sind für sie meist nur als Norm- und Traditionsbruch denkbar. Die Vorstellung, daß die schöpferische Hervorbringung literarischer Texte normativen Zwängen unterliegt, stößt bei ihr auf erheblichen Widerstand; allenfalls im Hinblick auf die Niederungen der massenhaft verbreiteten Unterhaltungsliteratur wird sie bereitwillig zugelassen. So verwundert es denn nicht, im Register einer neueren Schrift über „Poetik und Linguistik“4 zwar den Begriff der „Normdurchbrechung“, nicht jedoch den der „Norm“ selbst zu finden. Das ist symptomatisch für eine sowohl literarische als auch literaturwissenschaftliche Bewußtseinstradition, der Normen nur so weit

${ }^{3}$ Friedrich Scblegel, Schriften zur Literatur. Hrsg. von Wolfdietrich Rasch (München 1970) 38.

4 Rolf Kloepfer, Poetik und Lingustik. Semiotische Instrumente (München 1975). 
wichtig erscheinen, als sie von Texten verletzt werden. Die künstlerische Norm, so formulierte es Jan Mukařovský, bildet ,in der Regel nur den Hintergrund für den unaufhörlich gegen sie gerichteten Verstoß“. Und: „Die Geschichte der Kunst ist, wenn wir sie aus der Sicht der ästhetischen Norm betrachten, eine Geschichte der Auflehnung gegen die herrschenden Normen." Solchen Vorstellungen von Geschichte entsprechen literaturwissenschaftliche Wertungskonzepte, für die sich die ästhetische Qualitāt eines sprachlichen Kunstwerks aus seiner innovativen Distanz zu den „Erwartungen seines ersten Publikums ${ }^{\star 6}$ ergibt, für die also die Abweichung von geltenden Normen, um es paradox zu formulieren, selbst zur Norm geworden ist.

Das bināre Begriffspaar „Norm und Abweichung“ machte unlāngst Harald Fricke zum Titel und zum Zentrum einer "Philosophie der Literatur", die sich ebenfalls, ohne ein Bewußtsein davon zu zeigen, in den genieästhetischen Traditionen des 18. Jahrhunderts bewegt. Auch für Frickes Literaturphilosophie ist die Poesie das Reich der Freiheit, das sich den Notwendigkeiten sozialer Normierungen entzieht. „Soziale Normen“, wie der Autor sie verstanden wissen will, sind nicht in der poetischen, sondern nur in der normalen Sprache wirksam und verbindlich, und zwar in der Weise, daß normwidriges Sprachverhalten innerhalb einer Sprachgemeinschaft mit Sanktionen rechnen muß. Poetisches Sprechen (bzw. Schreiben) ist hingegen durch verschiedene Formen der Abweichung von der normalen und normierten Sprache gekennzeichnet, es folgt seinen eigenen Regeln, und das weithin autonom, ohne gravierende soziale Restriktionen ${ }^{7}$. Das Konzept entspricht der von Klopstock im Anschluß an Bodmer und Breitinger gegen Gottsched und Christian Weise hervorgehobenen Unterscheidung von Poesie und Prosa, die der Dichtung und dem Dichter eine autonome Sonderstellung gegenüber den geltenden Normen der prosaischen Vernunft zu sichern suchte. Die poetische Sprache ist nach Breitinger eine „Sprache der Leidenschaft". Und die Leidenschaften, so heißt es im zweiten Band seiner "Critischen Dichtkunst" von 1740, haben ,eine eigene Sprache und eine gantz besondere Art des Ausdrucks (...). Die Eigenschaft dieser Sprache bestehet demnach darinnen, daß sie in der Anordnung ihres Vortrags, in der Verbindung und Zusammensetzung der Wörter und Redensarten, und in der Einrichtung der Rede-Sätze sich an kein grammatisches Gesetze, oder logicalische Ordnung, die ein gesezteres Gemüthe erfordern, bindet."

Ich möchte im folgenden die von der Genieästhetik hervorgebrachten und von Literarhistorikern bis heute vielfach reproduzierten Varianten dichotomischer Gegenüberstellungen von normgebundener Heteronomie und normabweichender Autonomie in drei verschiedenen, doch aufeinander aufbauenden Thesen und Argumentationsschritten korrigieren oder zumindest modifizieren: zum einen mit Erinnerungen

${ }^{5}$ Jan Mukarorskj; Ästhetische Funktion, Norm und ästhetischer Wert als soziale Fakten, in:Jan Mukarotsky, Kapitel aus der Asthetik (Frankfurt am Main 1970) 38 und 46.

${ }^{6}$ Hans Robert Jauß, Literaturgeschichte als Provokation (Frankfurt am Main 1970) 177.

7 Harald Fricke, Norm und Abweichung. Eine Philosophie der Literatur (München 1981).

${ }^{8}$ Jobann Jacob Breitinger, Critische Dichtkunst. Faksimiledruck nach der Ausgabe von 1740, Bd. 2 (Stuttgart 1966) 354. 
an einige bereits bekannte Widersprüche und Fehleinschātzungen der Genieāsthetik, zum anderen im Rückgriff auf sozialwissenschaftliche Normtheorien und zum dritten mit Hilfe sozialgeschichtlicher Modernisierungstheoreme, die in besonderem Maße dazu geeignet sein könnten, eine Basis für das interdisziplinäre Gespräch zu schaffen.

1. Die enge Bindung der Literatur an die Rbetorik und Regelpoetik in Barock und Frübaufklärung bedeutete nicbt, daß literarische Texte durch Vorschriften (praecepta) und vorbildliche Muster (exempla), programmiert' waren und dem Autor keine Individualitätsspielräume offenstanden. Umgekebrt war die genieästhetische Programmatik und Praxis keineswegs frei von normativen Abhängigkeiten.

Daß der ,rhetorische Grundzug' der Barockliteratur vor allem in den auf Wirkungen angelegten Intentionen beruht und sich nicht auf normative Schreibanweisungen reduzieren läßt ${ }^{9}$, daß einzelne Texte nicht aus bestimmten Lehrbuchregeln ableitbar sind, sondern daß „die in den Rhetoriken und Poetiken niedergelegte Theorie nur den allgemeinsten Rahmen darstellt, der das Einzelne nur sehr bedingt präjudiziert" ${ }^{10}$, hat Wilfried Barner in seiner grundlegenden Arbeit zur „Barockrhetorik“ nachdrücklich betont und begründet. Gerade auch die Differenzen von, grob gesprochen, klassizistischer Regelpoetik und manieristischer Praxis zeigen, wie wenig es dem noch so subtil ausgearbeiteten System von Vorschriften gelungen sei, die literarische Elaboration festzulegen ${ }^{11}$. Darüber hinaus gilt es, der Bedeutung der „exempla“ und der „imitatio“ in der Disziplin der Rhetorik angemessen Rechnung zu tragen, ohne deren Kenntnis sowohl der Einzeltext als auch die Pluralität der Stile und der an sie gekoppelten Traditionen sich einem angemessenen Verständnis entziehen ${ }^{12}$.

Auch die starke Bindung der literarischen Praxis an die Imitation vorbildlicher $\mathrm{Mu}-$ ster ist nicht von vornherein als ein individualitätswidriges Phānomen zu verstehen. Christian Weises „Curiöse Gedancken von der Imitation“ (1698) enthalten eine aufschlußreiche Passage, die zur Ausbildung eines eigenen Stils ausdrücklich auffordert und der individuellen Begabung des einzelnen ausdrücklich Rechnung trägt; denn es wird ,sich kein Mensch zu einem Stylo (...) zwingen lassen/ dazu er keine sonderliche Kunst verspüret". Zwar sind in diesem Rhetorikkonzept Imitationsübungen nach wie vor unumgānglich, doch dienen sie dazu, „daß ein jedwedes Ingenium den Stylum möchte kennen lernen/ dazu sein naturel am meisten incliniret“. Zwar muß der Gelehrte „etliche Periodos schreiben können/ wie Salustius, etliche wie Livius, etliche wie Caesar, etliche gar wie Seneca, oder Tacitus“, gleichwohl bleibt er ,ausser diesem Zwange bey seiner Freyheit“ und kann „nach Anleitung eines angenehmen Vorgängers“ so singen, „wie ihm also der Schnabel gewachsen ist“" ${ }^{\text {“3 }}$. Man könnte versucht

9 Wilfried Barner, Barockrhetorik. Untersuchungen zu ihren geschichtlichen Grundlagen (Tübingen 1970) 72 ff.; im folgenden zitiert: Barner, Barockrhetorik.

${ }^{10}$ Ebd. 453.

11 Ebd. $63 \mathrm{f}$.

12 Ebd. 59 ff.

${ }^{13}$ Christian Weise, Curiöse Gedancken von der Imitation ... (Leipzig 1698) 304 ff. 
sein, solche Sātze als zukunftsweisende Belege für die Emanzipation des bürgerlichen Individuums von Fixierungen an Traditionen und Normen zu lesen, und dabei auf den literaturgeschichtlichen Ort Christian Weises als einer Figur des Übergangs vom Barock zur Aufklärung verweisen. Diese Einschätzung entspräche indes ganz der genieästhetischen Kritik an einer regelfixierten Rhetorik, die der Individualität angeblich keinen Spielraum ließ. Weise knüpfte mit seiner Forderung, aus der Pluralität zugänglicher Stilexempla das dem eigenen Naturell gemäße herauszufinden und im Durchgang durch die Nachahmung zur Freiheit eines individuellen Stils zu gelangen, an Argumente aus den humanistischen Debatten über die kanonische Geltung von Ciceros Stil an, in denen vor allem Angelo Poliziano und Erasmus das Recht auf individuelle Differenz zu den kanonisierten Autoren der Antike reklamierten: „Man sagt mir, daß ich mich nicht wie Cicero ausdrücke, obwohl ich ihn so lange studiert habe. Aber ich bin nicht Cicero und gerade von Cicero habe ich gelernt, ich selbst zu sein." ${ }^{14}$ So Poliziano. Nach Hans Joachim Langes einschlägiger Arbeit zu dem Thema schuf Erasmus „das Nachahmungskonzept der ,aemulatio“, mit deren Hilfe der Normanspruch eines Kanonautors erschüttert, die glāubige Befolgung der Normgebote lācherlich gemacht und diese selbst als willkürlich entlarvt werden konnten, mit deren Hilfe schließlich der Drang nach der eigenen, unverwechselbaren, einmaligen und dem Zeitgeist entsprechenden Ausdrucksgebärde gerechtfertigt und aus dem Geist der Überlieferung selbst hergeleitet werden konnte ${ }^{\text {"15 }}$. Die Argumentation der AntiCiceroneaner war den Barockrhetorikern bekannt, doch bevorzugten ihre Imitationslehren zur Abgrenzung von der bloßen Kopie angesehener Vorbilder einen etwas anderen Argumentationstyp, der den individuellen Spielraum des sprachlichen Verhaltens weniger in der Selektion als in der Kombination bewährter Muster ansiedelte. Harsdörffers „Poetischer Trichter" bediente sich des traditionsreichen und in diesen Zusammenhängen überaus beliebten Bienengleichnisses: „Wir wollen den Bienen nachahmen/ und was wir in unterschiedne Büchern gelesen/ unterschiedlich bemerken; nochmals aber mit verständigem Fleiß zusammen mischen/ daß ob man gleich wissen kann/ woher es genommen/ jedoch etwas anders daraus gemachet worden/als es gewesen." 16 Birken schrieb in seiner "Teutschen Rede- bind- und Dicht-Kunst" āhnlich: „Man darf wol borgen/ oder übersetzen: aber man muß denjenigen nennen/ von dem man geborget/ und nicht zum Diebe werden. Man muß entnehmen/ wie die

${ }^{14}$ Zitiert nach Volker Sinemus, Poetik und Rhetorik im frühmodernen deutschen Staat. Sozialgeschichtliche Bedingungen des Normenwandels im 17. Jahrhundert (Göttingen 1978) 115; im folgenden zitiert: Sinemus, Poetik und Rhetorik.

is Hans Joacbim Lange, Aemulatio Veterum sive de optimo genere dicendi. Die Entstehung des Barockstils im XVI. Jahrhundert durch eine Geschmacksverschiebung in Richtung der Stile des manieristischen Typs (Frankfurt 1974) 119f.; vgl dazu die kritischen Einschrānkungen von Sinemus, Poetik und Rhetorik, 115 und 327.

${ }^{16}$ Georg Pbilipp Harsdörffer, Poetischer Trichter. Die Teutsche Dicht- und Reimkunst ohne Behuf der Lateinischen Sprache in VI. Stunden einzugiessen. III. Teil (Nürnberg 1653) 54 (Hervorhebung von mir); zur Tradition des "Bienengleichnisses“ Jürgen von Stackelberg, Das Bienengleichnis. Ein Beitrag zur Geschichte der literarischen Imitatio, in: Romanische Forschungen 68 (1956) 271-293; Literaturhinweise zur Imitationslehre bei Barner, Barockrhetorik, 59 (Anm. 98). 
Bienen ihr Honig aus den Blumen. Man muß das Gehirn zum guten Magen machen/ der die Speise/ nicht wie er sie empfangen wieder herauskotze/ sondern verdeue und in eignen Nahrung-Saft verwandle. ${ }^{17}$ Gottsched akzentuierte später in dem der Imitatio geltenden Teil seiner „Ausführlichen Redekunst“ die Forderung nach der originären Eigenleistung in einer Weise, die man ihm kaum zutraut: „Aber wenn man selbst die Feder ansetzet, dann muß man alle andere Bücher der guten Stilisten bey Seite legen, und alles aus seinem Kopfe schreiben." Und: „Man muß sich vielmehr selbst vornehmen, ein Original zu werden, und anderen zum Muster dienen. Denn wer dazu nicht Muth genug hat, der wird selten etwas großes unternehmen." ${ }^{18}$

Man findet schon im 17. Jahrhundert erstaunliche Äußerungen, die sich satirischkritisch von regelpoetischen Anweisungen, bloßer Gelehrsamkeit und platter Imitation distanzieren ${ }^{19}$ und damit einmal mehr zeigen, wie wenig die Genieästhetik mit ihren Verdikten gegen Rhetorik und Regelpoetik dem Selbstverstāndnis des Barock und der Frühaufklärung gerecht wurde. Doch auch ihre Selbsteinschätzung war voller Selbsttäuschungen. Die alle Reglementierungen von sich weisenden "Genies“ antizipierten die Sanktionen der Zensur durch entsprechende Umarbeitungen ihrer Stücke ${ }^{20}$. Hamanns und Herders Kritik der Gelehrsamkeit blieb selbst in hohem Maße gelehrsam; die Kritik rhetorischer Kunstausübung im Namen vernünftiger oder empfindsamer Natürlichkeit blieb rhetorisch; die pietistische, gegenbarocke Forderung nach ungekünsteltem, authentischem Herzensausdruck bediente sich traditionsreicher rhetorischer Argumentationsmuster ${ }^{21}$; der Spott auf die nach Rezepten gemachte Dichtung schloß nicht aus, daß man selbst dem Geniepoeten genaue Anweisungen bis in den Satzbau hinein gab $^{22}$, die Kritiker der Nachahmung ahmten selber exzessiv nach. Ein Paradigma der Geniedichtung, Goethes Ode „Wandrers Sturmlied“, ist zugleich das Musterbeispiel eines nach gelehrt-rhetorischen Anweisungen durchkonstruierten Textes ${ }^{23}$. Der Begriff des „Kunstrichters“ wurde in einer Zeit zum Schimpfwort, in dem sich die Literaturkritik erst eigentlich institutionalisierte ${ }^{24}$.

\footnotetext{
${ }^{17}$ Sigmund von Birken, Teutsche Rede- bind- und Dicht-Kunst ... (Nürnberg 1679) 178 (Hervorhebung von mir).

${ }^{18}$ Jobann Cbristopb Gottsched, Ausführliche Redekunst (Leipzig $\left.{ }^{4} 1750\right) 350$ (Hervorhebung von mir).

19 Beispiele dafür in dem Kapitel „Poetik und Rhetorik“ bei Ferdinand van Ingen, Vanitas und Mernento Mori in der deutschen Barocklyrik (Groningen 1966) 39, $43 \mathrm{f}$.

${ }^{20} \mathrm{Vgl}$. das Kapitel zur Zensur in Helmuth Kiesel/Paul Münch, Gesellschaft und Literatur im 18. Jahrhundert. Voraussetzungen und Entstehung des literarischen Markts in Deutschland (München 1977) 120 f.; im folgenden zitiert: Kiesel/Münch, Gesellschaft und Literatur.

${ }^{21}$ Vgl. Wolfgang Martens, Hallescher Pietismus und Rhetorik. Zu Hieronymus Freyers „Oratoria“, in: Internationales Archiv für Sozialgeschichte der Literatur 9 (1984) 22-43 und die dort in Anm. 10 und 11 genannten Arbeiten von Reinhard Breymayer.

${ }^{22} \mathrm{Vgl}$. Schmidt, Geschichte des Genie-Gedankens, 94.

${ }^{23}$ Ebd. $199 \mathrm{ff}$.

${ }^{24}$ Vgl. Klaus L. Bergbabn, Von der klassizistischen zur klassischen Literaturkritik 1730-1806, in: Geschichte der deutschen Literaturkritik (1730-1980). Hrsg. von Peter Uwe Hobendabl (Stuttgart 1985) $10-75$.
} 
Das heißt nicht, daß sich im 18. Jahrhundert die literarische Theorie und Praxis nicht veränderten, doch sind diese Veränderungen durch binäre Begriffsdistinktionen wie thetorisch und antirhetorisch, regelfixiert und regelenthoben, traditionsgebunden und traditionsfrei, normabhāngig und normabweichend, heteronom und autonom usw. unzulānglich beschrieben. Vielmehr ist die Rhetorikkritik im 18. Jahrhundert eine Kritik bestimmter Ausprägungen der Rhetorik, denen andere vorgezogen werden. Traditionen werden nicht grundsätzlich abgelehnt, sondern uminterpretiert oder durch andere ersetzt ${ }^{2 s}$. Exempla behalten ihre stilbildende Attraktivität, doch die Genieästhetik favorisiert ihre eigenen: Homer, Pindar, Shakespeare. Der Kritiker hört nicht auf zu richten, aber er richtet nach partiell verānderten Kriterien. Kurzum: Literarische Normen verlieren nicht grundsätzlich ihre Verbindlichkeit, sondern sie wandeln sich.

Beruht also das Individualitāts- und Originalitätspathos der Genieästhetik auf bloBen Selbsttāuschungen, denen auch die Literaturwissenschaft lange Zeit aufgesessen ist? Ist, wie Pierre Bordieu in seiner „Soziologie der symbolischen Formen“ fragte, „die ästhetische Revolution, die in der Theorie von der höheren Wahrheit der Kunst und des autonomen Genies Ausdruck findet, als die simple ideologische Kompensation der Bedrohung anzusehen, die mit der industriellen Gesellschaft und durch die Industrialisierung der Intellektuellengesellschaft auf der Autonomie des künstlerischen Schaffens und der unersetzlichen Einzigartigkeit des ,Gebildeten' lastet? ${ }^{626}$

Es hieße, ein polemisch verzerrtes Bild vom gegenwärtigen Stand der Literaturwissenschaft zu zeichnen, wenn man ihr immer noch pauschal den unreflektierten Nachvollzug genieāsthetischer Positionen unterstellte. Deren Spuren sind zwar heute nach wie vor deutlich in literaturwissenschaftlichen Konzepten ablesbar, doch spätestens seit Ende der sechziger Jahre haben sich mittlerweile etablierte Gegenkonzepte herausgebildet, die dem Begriff des autonomen Subjekts oder Individuums in dem Maße skeptisch bis ignorant gegenüberstehen wie vormals die Geisteswissenschaften dem der Norm. Nicht nur aus Gründen methodologischer Reinlichkeit tendieren sozialgeschichtliche und systemtheoretische, strukturale und diskursanalytische Methoden zu einer ausgesprochenen Individualitätsfeindlichkeit. Das einzelne Werk und die Persönlichkeit des Autors lösen sich ihnen auf in gesellschaftlichen Machtkonstellationen, sozialen Systemen, sprachlichen Strukturen, poetischen Codes, Diskursordnungen oder gleichsam maschinellen „Aufschreibesystemen“. Es ist schon ein bemerkenswertes wissenschaftsgeschichtliches Phänomen, mit welcher affirmativen Genugtuung heute häufig einer sich selbst autonom dünkenden Literatur strukturelle und funktio-

${ }^{25}$ Zur Problematik des Traditionsbegriffs im Hinblick u. a. auf die „Epochenwende“ im 18. Jahrhundert vgl. jetzt den grundlegenden Beitrag von Wilfried Barner, Über das Negieren von Tradition. Zur Typologie literaturprogrammatischer Epochenwenden in Deutschland, in: Epochenschwelle und Epochenbewußtsein. Poetik und Hermeneutik XII. Hrsg. von Reinbart Herzog und Reinhart Koselleck (München 1987) 3-51.

${ }^{26}$ Pierre Bourdieu, Zur Soziologie der symbolischen Formen (Frankfurt am Main 1970) 81; im folgenden zitiert: Bourdieu, Soziologie symbolischer Formen. 
nale Abhängigkeiten nachgewiesen werden, wie sie lange Zeit, mit negativer Bewertung, der von höfischen Anpassungszwängen und rhetorischen Regeln angeblich programmierten Literatur des Barock unterstellt wurden.

2. Handlungs, norm- und rollenthearetische Ansätze vor allem aus dem Umkreis des "Symbolischen Interaktionismus" bieten beute der Literaturgeschicbtsscbreibung Anregungen zur interdisziplinären Reflexion über das Verbältnis von Norm und Autonomie, die dazu geeignet sind, gängige Dicbotomisierungen zu überwinden.

Die literaturwissenschaftliche Adaptation sozialwissenschaftlicher Handlungs-, Norm- und Rollenbegriffe erscheint auf den ersten Blick ausgesprochen rhetorik- und barockfreundlich, doch dem angemessenen Zugang zum genieästhetischen Individualitäts- und Autonomiebegriff eher abträglich. Indem sie den Akt literarischen Schreibens (sowie alle anderen Umgangsformen mit Literatur) als ein sprachliches Rollenspiel begreift, das an den Erwartungen der Adressaten orientiert ist, greift sie auf eben jene Theatermetaphorik zurück, die ein konstitutiver Bestandteil barocker Bildlichkeit und für das Verständnis der Barockrhetorik grundlegend ist ${ }^{27}$. „Wir alle spielen Theater “ lautete der Titel einer berühmten, dem Symbolischen Interaktionismus nahestehenden Schrift des amerikanischen Soziologen Erving Goffman ${ }^{28}$. Im Anschluß an symbolisch-interaktionistische und sprechakttheoretische Konzepte aus der sprachanalytischen Philosophie ${ }^{29}$ läßt sich der literarische Schreibakt als eine soziale Handlung begreifen, die auf das Handeln anderer Menschen (potentiell aller, die mit Literatur etwas ,tun') intentional bezogen ist. Der Autor antizipiert mehr oder weniger bewußt, wie jeder, der sich sprachlich äußert, in der Phantasie die Reaktion des Angesprochenen. George Herbert Mead, der ,Protagonist' des „Symbolischen Interaktionismus", nannte diesen Antizipationsvorgang „Rollenübernahme“ (,taking the role of the other"): Im Akt des Sprechens versetzt der Sprecher sich in die Rolle des Angesprochenen und vermag so die eigenen Äußerungen im Hinblick auf die vermuteten Reaktionen des Partners zu steuern ${ }^{30}$.

Mit einem knapp gehaltenen Exkurs zu Norm- und Rollenbegriffen und zu ihrer Übertragbarkeit auf literarische Interaktionsprozesse erläuterte ich einige theoretische Voraussetzungen für die abschließenden Überlegungen über die verānderten Individualitätsspielräume in der modernisierten Literaturgesellschaft des 18. Jahrhunderts ${ }^{31}$.

${ }^{27} \mathrm{Vgl.} \mathrm{Barner,} \mathrm{Barockrhetorik,} 86$ ff.; zum Verhältnis von soziologischem und ästhetischem Rollenbegriff Hans Robert Jauß, Ästhetische Erfahrung und literarische Hermeneutik (Frankfurt am Main 1982) 221-231.

${ }^{28}$ Erving Goffman, Wir alle spielen Theater. Die Selbstdarstellung im Alltag (München 1969).

29 Siehe die bei $A n z$, Soziologie literarischer Normen, in Anm. 17-19 genannten Titel von Austin, Searle, Mead, Joas und Habermas.

${ }^{30}$ Siehe Hans Joas, Die gegenwàrtige Lage der soziologischen Rolientheorie (Frankfurt 1973) $36 \mathrm{f}$.

${ }^{31}$ Das Folgende übernehme ich, geringfügig überarbeitet, aus $A n z$, Soziologie literarischer Normen, 133 ff.; vgl. dazu den umfassenderen Beitrag von Renate von Heydebrand, Wertung, literarische, in: Reallexikon der deutschen Literaturgeschichte. Bd. 4, letzte Lieferung. Hrsg. von Klaus Kanzog und Achim Maser (Berlin, New York 1984) 828-871. 
Soziales (auch sprachliches und literarisches) Handeln ist u.a. konditioniert durch ein Geflecht wechselseitiger Erwartungen und Ansprüche, durch soziale Normen. die den Aktoren einen Orientierungsrahmen geben. Normen sind dabei dem Handeln (auch Schreiben) nicht immer schon statisch vorgegeben, sondern sie entstehen, stabilisieren und verändern sich in langfristigen Interaktionsprozessen (z. B. zwischen Autoren, Literaturvermittlem und Lesern). Normen können jederzeit, wenn auch nicht folgenlos, übertreten werden, doch gehören sie zu den Bedingungen der Möglichkeit kontinuierlicher zwischenmenschlicher (auch literarischer) Beziehungen. Sie begrenzen, wie Heinrich Popitz formuliert, „die Willkür in den Beziehungen von Menschen zueinander“ und „bewirken, daß Menschen sich mit einiger Sicherheit und Dauerhaftigkeit aufeinander einstellen können“32. Sie reduzieren in psychisch entlastender Weise die Komplexităt der Verhaltensmöglichkeiten, die man vom Partner und sich selbst in sozialen Beziehungen erwarten kann ${ }^{33}$. „Soziale Normen beschränken“, so Hans Peter Dreitzel, „die Möglichkeit der Bedürfnisbefriedigung, damit überhaupt Bedürfnisse befriedigt werden können."34 Ein normenloser Zustand hätte zwar, und das kann auch für die Produktion und Rezeption literarischer Werke gelten, den entlastenden Vorzug einer gleichsam anarchischen Freiheit, es gibt indes einen sozialwissenschaftlichen (von Emile Durkheim eingeführten) Begriff, der die Kehrseite dieser Ungebundenheit beschreibt: „Anomie“. Den damit bezeichneten Zustand der Normenlosigkeit sieht man gekoppelt mit einer psychisch belastenden Orientierungslosigkeit und Vereinzelung der Subjekte.

An welchen Normen sich jemand, der eine soziale Handlung vollzieht (der Aktor oder auch Autor), orientiert, hängt davon $a b$, in welcher Situation und Rolle er handelt bzw. welchen Typ einer sozialen Handlung er ausführt. Lehrer, Verkäufer, Wissenschaftler, Lektoren, Kritiker oder Dichter haben in ihrem rollentypischen Sprachverhalten jeweils unterschiedliche Normkomplexe zu berücksichtigen, was freilich die Existenz allgemeinerer, rollenübergreifender Normen nicht ausschließt. Rollentheoretische Analysen von Verhaltensnormen differenzieren diese nach verschiedenen Typen sozialer Interaktion. Auch der Schreibakt des Autors ist in diesem Sinne eine Rollenhandlung, doch ist auch sie noch weiter spezifizierbar: in die Rolle des Komödien- oder Tragödiendichters, des Erzählers oder des Lyrikers usf. Aus normensoziologischer Perspektive läßt sich das Schreiben im Rahmen bestimmter Gattungen als institutionalisiertes Rollenspiel analysieren ${ }^{35}$, das sich jeweils deutlich unterschiedenen Erwartungen gegenübersieht.

Unter Rollennormen verstehe ich im folgenden einen Komplex von Erwartungen, die ein Rollenspieler von anderen an sein Verhalten gerichtet sieht und bei deren Miß-

32 Heinrich Popitz, Soziale Normen, in: Europäisches Archiv für Soziologie 2 (1961) 187.

${ }^{33} \mathrm{Vgl}$. Niklas Lubmann, Normen in soziologischer Perspektive, in: Soziale Welt 20 (1969) $28-48$.

${ }^{34}$ Hans Peter Dreitzel, Die gesellschaftlichen Leiden und das Leiden an der Gesellschaft. Vorstudien zu einer Pathologie des Rollenverhaltens (Stuttgart 1972) 224; im folgenden zitiert: Dreitzel, Die gesellschaftlichen Leiden.

${ }^{35}$ Vgl. Wilhelm Voßkamp, Gattungen als literarisch-soziale Institution, in: Textsortenlehre Gattungsgeschichte. Hrsg. von Walter Hinck (Heidelberg 1977) 30 ff. 
achtung er mit negativen Sanktionen rechnen muß. Normen lassen sich als Verbote oder Gebote formulieren. Rollen- $b z w$. Normadressat ist der, an den die Erwartungen gerichtet sind. Rollen-bzw. Normsender sind diejenigen Personen oder Institutionen, von denen die Erwartungen und zum Teil auch die potentiellen Sanktionen ausgehen. Sie lassen sich nach unterschiedlichen Bezugsgruppen klassifizieren. Autoren z. B. sehen sich beim Schreiben mit den Erwartungen verschiedener Publikumsgruppen konfrontiert: Mäzenen, Lektoren und Verlegern, Freunden, literarischen Schulen, Literaturkritikern, Zensoren usw. Kann der Autor die eventuell stark divergierenden Erwartungen der für ihn relevanten Bezugsgruppen nur schwer oder gar nicht integrieren (der Autor z.B. die Erwartungen seines Freundeskreises und die des Verlags oder seines Zielpublikums), dann steht er in einem Intra-Rollenkonflikt, in einem Inter-Rollenkonflikt dann, wenn er seine vielfältigen Rollenverpflichtungen (z. B. als Autor, Berufstätiger oder Familienvater) nicht oder nur schwer miteinander vereinbaren kann. Rollenkonflikte gefährden die Icb-Identität.

Die Verbindlichkeit von Normen wird durch Sanktionen signalisiert und gestützt. Positive Sanktionen bestātigen bestimmte (hoch gewertete) Verhaltensweisen (die literarischen des Autors z. B. in Form von lobenden Rezensionen, Preisverleihungen, Verkaufserfolgen usw.), negative Sanktionen negieren sie (z. B. in Form von Freiheits- oder Geldstrafen, Publikationsverboten, Exkommunikation, Pathologisierungen, abwertenden Rezensionen, Entzug der Anerkennung, Ignoranz, Ausschluß aus der Künstlergruppe). Nach Art der Sanktionspraktiken lassen sich verschiedene Verbindlichkeitsgrade von Normen unterscheiden. Muß-Erwartungen werden in der Regel durch negative, sehr scharfe Sanktionsandrohungen gestützt (sie betreffen Literatur wohl nur auf ihrer thematischen Ebene, vor allem ihre zensurgefährdete Haltung zu Politik, Religion, Sexualität und Gewalt). Negative, wenn auch weniger strenge Sanktionen (etwa „Verrisse“ durch die Kritik) überwiegen auch bei der Mißachtung von Soll-Erwartungen. Bei der Durchbrechung von Kann-Erwartungen sind dagegen nicht derart einschneidende negative Folgen zu erwarten. Sie lassen sich auch als Ideal-Normen begreifen, denen zu entsprechen keineswegs selbstverständlich ist, sondern eine Leistung, die in Form von positiven Sanktionen ausdrücklich honoriert werden kann.

Die Übertragung der bis hierher erläuterten Begriffe auf literarisches Handeln scheint noch wenig geeignet, das oben kritisierte dichotomische Denken zu überwinden. In der mit ihnen verbundenen Perspektive erscheint der Autor, wie in manchen mittlerweile obsolet gewordenen Vorstellungen der Rezeptionsforschung, als bloßes Vollzugsorgan von Leser- bzw. Bezugsgruppenerwartungen oder, im Falle eigenwilliger Normabweichung, als ein dysfunktionales Störelement im System literaturgesellschaftlicher Interaktionsprozesse. Dies entspräche etwa dem Stand der Rollentheorie, wie sie Ralf Dahrendorfs klassische Schrift „Homo Sociologicus“ reprāsentiert. In der Konzeption Dahrendorfs verhalten sich die Rollenspieler weithin konformistisch-reaktiv. Das Konstrukt des „homo sociologicus“ und die Idee des autonomen Individuums stehen sich unversöhnlich schroff gegenüber ${ }^{36}$. Entsprechend den eingangs skiz-

${ }^{36}$ Ralf Dabrendorf, Homo Sociologicus. Ein Versuch zur Geschichte, Bedeutung und Kritik der Kategorie der sozialen Rolle (Köln, Opladen ' 1970 ). 
zierten Denk- und Begriffstraditionen kann sich die Freiheit und Originalitāt einer Person dann nur in der gesellschaftlich unerwünschten und destruktiven Mißachtung von Rollenerwartungen bezeugen.

Demgegenüber, und das richtet sich mit Einschränkungen auch gegen die Systemtheorie Parsons und ihre Weiterentwicklungen bis hin zu Luhmann ${ }^{37}$, haben Handlungs- und Normtheorien insbesondere aus dem Umkreis des „Symbolischen Interaktionismus “ Konzepte entwickelt ${ }^{38}$, die mir zumindest für die literaturwissenschaftliche Aneignung soziologischer Rollen- und Normbegriffe unter dem Kriterium der Gegenstandsadäquatheit sehr viel geeigneter erscheinen. In ihnen akzentuiert man die Bedeutung von Ich-Leistungen für das Rollenverhalten und verwendet dabei u.a. den Begriff der „Rollendistanz“ zur Kennzeichnung der Fähigkeit, ein flexibles, interpretierendes, reflektierendes oder auch selbstironisches Verhältnis zu vorgegebenen Normen einzunehmen. Ich-Leistungen sind in mehr oder weniger starkem Maße bei jeder Rollenhandlung gefordert. Zwar läßt sich die Wirksamkeit (bzw. Geltung) sozialer Normen an normentsprechenden, überindividuellen Verhaltensregelmāßigkeiten ablesen, doch ist das faktische Verhalten niemals durch Normen vollständig festgelegt. Individuen verhalten sich in der gleichen Rolle und gegenüber den gleichen Anforderungen oft sehr verschieden. Viele Bereiche des sozialen Handelns sind gar nicht normiert, andere nur schwach. Oft orientiert sich das soziale Handeln an „kulturell tradierten Werte( $n)^{\text {“, }}$, so Hans Peter Dreitzel, die „unspezifisch, vage und allgemein gehalten“" sind; „sie weisen dem Verhalten eher eine Richtung, als daß sie konkrete Vor-

${ }^{37}$ Einen Überblick über den Beitrag der klassischen Soziologie zum Thema "Soziale Ordnung und individuelle Autonomie" gibt das so betitelte Kapitel in Richard Münch, Theorie des Handelns. Zur Rekonstruktion der Beiträge von Talcott Parsons, Emile Durkheim und Max Weber (Frankfurt am Main 1982) 281-426; im folgenden zitiert: Münch, Theorie des Handelns. In dem durchgehenden Bemühen, Parsons gegen seine Kritiker zu verteidigen, hebt diese Schrift freilich die Differenzen zwischen Systemtheorie und Symbolischem Interaktionismus weitgehend auf. Luhmann ist auf das Thema ausführlicher eingegangen in: Niklas Lubmann, Soziale Systeme. Grundriß einer allgemeinen Theorie (Frankfurt am Main 1984) 346-376; im folgenden zitiert: Luhmann, Soziale Systeme. Luhmann überträgt hier die Theorie autopoietischer (d. i. sich selbst regulierender) Systeme auf das Problem der Individualität psychischer Systeme. Es bestehe kein Zweifel, „daß psychische Systeme autopoietische Systeme sind“ (S. 355). „Individualität" meint in diesem Zusammenhang die „zirkuläre Geschlossenheit“ (S. 358) eines Systems. „Autopoiesis des Bewußtseins ist mithin die faktische Basis der Individualitāt psychischer Systeme." (S. 359) Psychische Systeme sind in diesem Konzept immer schon als Individualitāten zu begreifen, für die andere Systeme (u. a. soziale) zur „Umwelt“ gehören. Die Abhängigkeiten der Systeme, die füreinander Umwelten bilden, beschreibt Luhmann unter dem Begriff der „Interpenetration“. Vgl. die Kritik an diesem Konzept von Jürgen Habermas, Der philosophische Diskurs der Moderne (Frankfurt am Main 1985) 426ff. Das neuzeitliche Individualitātspathos ist für Luhmann vornehmlich durch die forcierten Anstrengungen zur Selbstbeobachtung und -beschreibung charakterisiert. Es stelle sich dabei „die Frage, ob und unter welchen gesellschaftlichen Bedingungen ihm (dem Individuum, Th.A.) das Insistieren auf Individualităt als Selbstbeschreibung erlaubt oder gar aufgenötigt wird“. (S. 361)

${ }^{38}$ Siehe dazu besonders Dreitzel, Die gesellschaftlichen Leiden; Hans Joas, Praktische Intersubjektivităt. Die Entwicklung des Werkes von G. H. Mead (Frankfurt 1980); Jürgen Habermas, Theorie des kommunikativen Handelns, Bd. 1 (Frankfurt am Main 1981) 11 ff. 
schriften enthielten." ${ }^{39}$ Normen bieten oft nur abstrakte Vorgaben, deren Konkretisierung für das Verhalten in einer bestimmten Situation dem Ich einige interpretatorische und kreative Leistungen abverlangt.

Individualitäts- und Originalitätsansprüche lassen sich demnach keineswegs nur durcb Abweichungen von Normen realisieren, sondern auch in der kreativen Interpretation von Normvorgaben und in der Ausgestaltung des freien Spielraums, den Normen in mebr oder weniger starkem Maße offenlassen.

Dreitzel hat in seiner erstmals 1968 erschienenen und meines Erachtens bis heute unübertroffenen Schrift zur Rollentheorie Normen nach den von ihnen zugelassenen oder sogar miterwarteten Ich-Leistungen unterschieden und typisiert. „Dem Soldaten etwa ist sein Tun und Lassen bis ins Kleinste vorgeschrieben, die erforderlichen IchLeistungen waren ursprünglich gleich Null, und erst mit der zunehmenden Technisierung des Kriegswesens wird etwas mehr Selbständigkeit von ihm erwartet. Die Rolle des akademischen Lehrers dagegen setzt sich von vornherein aus sehr unterschiedlichen Erwartungen zusammen, die überdies einen breiten Spielraum für die eigene Initiative und Ausgestaltung lassen, gewissermaßen eine Leerstelle, deren Ausfüllung aber mit zu den Rollenerwartungen gehört. ${ }^{\text {“40 }}$ Im Hinblick auf die geforderten IchLeistungen unterscheidet Dreitzel, und das gibt zu einer normtheoretischen Reformulierung der Differenzen zwischen Regelpoetik und Genie- oder Autonomieästhetik wertvolle Anregungen, grob zwischen Vollzugsnormen, Qualitātsnormen und Gestaltungsnormen: „Die Vollzugsnormen lassen den Ich-Leistungen nur einen sehr engen Spielraum; es geht statt dessen um die gehorsame Einordnung in ein relativ dichtes Netz von Regeln. Bei den Qualitātsnormen steht die Aufgabe und ihre Bewältigung im Vordergrund; Ich-Leistungen und Regeln der Ausführung halten sich die Waage. Das Hauptproblem ist hier die Legitimität adäquater Mittel und Normen. Bei den Gestaltungsnormen stehen dagegen die Ich-Leistungen im Vordergrund, weil der individuelle Stil und die persönliche, möglicherweise neuartige Problemlösung gerade das sind, was die Rolle vorschreibt. “41

In literarischen Kulturen divergiert der Spielraum für Ich-Leistungen historisch und gattungsspezifisch. Der Weg von Gottscheds Poetik zu Baumgartens Ästhetik etwa wäre in dieser Perspektive auch eine Entwicklung zu abstrakteren Normen. Die Differenzen zwischen Barockrhetorik und Geniedichtung wären nicht mehr mit dem Gegensatz normgebunden und nicht normiert zu beschreiben, sondern als Unterschiede von Normtypen, und zwar, folgt man der Terminologie Dreitzels und setzt man die schon im 17. Jahrhundert geforderten Ich-Leistungen nicht zu niedrig an, zwischen „Qualitātsnormen“ (nicht „Vollzugsnormen“!) und „Gestaltungsnormen“. Individualität, Innovation und Originalităt sind in dieser Sichtweise Werte, die nicht jenseits aller Normen liegen, sondern konstitutiver Bestandteil dessen sind, was von einem Autor unter veränderten sozialen Bedingungen in einer modernisierten Literaturgesellschaft erwartet wird.

${ }^{39}$ Dreitzel, Die gesellschaftlichen Leiden, 112.

40 Ebd. 117.

41 Ebd. 118. 
3. Im Rabmen gesamtgesellschaftlicher Modernisierungsprozesse differenziert sich die deutsche Literaturgesellschaft im 18. Jabrbundert zu einem relativ autonomen System aus. Der relativen Autonomie der modernisierten Literaturgesellschaft entsprechen erweiterte Individualitätsspielräume der in ihr Handelnden.

Die Ausbildung der Genieāsthetik sieht man gewöhnlich an sozialgeschichtliche Entwicklungsprozesse gebunden, in denen sich der Autor der Abhängigkeiten vom Hof und von der Gelehrtenzunft entledigt und zum „freien Schriftsteller“ emanzipiert. „Der Weg vom Untertanen zum freien, unabhängigen, nur auf seine eigene Leistungskraft gestellten Bürger - das ist auch der Weg vom Hofdichter und vom poetisch kompetenten Mitglied der Gelehrtenzunft zum autonomen, sein Selbstbewußtsein ganz auf seine Schöpferkraft gründenden Dichter. Die Genie-Proklamationen, in denen die literaturästhetische Entwicklung des 18. Jahrhunderts gipfelt, sind Manifestationen des unabhängig gewordenen, oft genug auch nur Unabhängigkeit ersehnenden, auf seine eigenen produktiven Energien stolzen bürgerlichen Menschen, der keine andere Autoritāt mehr anerkennt. “42 Nach der skeptischen und desillusionierenden Variante dieses Erklärungsmodells, die hier beiläufig anklingt („Unabhängigkeit ersehnend“), treten für den Schriftsteller an die Stelle der Abhängigkeiten vom Hof die Abhängigkeiten vom literarischen Markt: „In jedem Fall täuschte aber das Epitheton ,frei über die realen Marktabhängigkeiten des Schriftstellers hinweg und evozierte völlig zu Unrecht die Vorstellung autonomer Kunstproduktion sowie ungehinderter Entfaltung aller intellektuellen Krāfte und ästhetischen Neigungen." $\$ 3$

Im Rückgriff auf rollentheoretische Begriffe und sozialwissenschaftliche Modernisierungstheoreme möchte ich abschließend eine demgegenüber modifizierte Sichtweise der angesprochenen sozialgeschichtlichen Veränderungen zur Diskussion stellen $^{44}$. Viele der von sozialgeschichtlich orientierten Literarhistorikern seit den sechziger Jahren vorgelegten Materialien und Stichworte zur Geschichte der Publikumsgeschichten, Lesegesellschaften und Bibliotheken, des Buchmarktes, des Verlagswesens oder der Zeitschriften im 18. Jahrhundert ${ }^{45}$ lassen sich im Rahmen sozialwissenschaftlicher Modernisierungstheorien systematisieren. Die unter diesem Etikett seit etwa 1960 entwickelten Theorien über soziale und politische Entwicklungen verschiedenster Art waren in den Gegenüberstellungen von Merkmalen der Traditionalitāt und Modernität anfänglich ebenfalls den eingangs kritisierten dichotomischen Konstruk-

42 Schmidt, Geschichte des Genie-Gedankens, 12

(3) Kiesel/Münch, Gesellschaft und Literatur, 88.

44 Zur literaturwissenschaftlichen Adaption system- und modemisierungstheoretischer Debatten vgl. den Band: Zur theoretischen Grundlegung einer Sozialgeschichte der Literatur. Ein struktural-funktionaler Entwurf. Hrsg. im Auftrag der Münchener Forschergnuppe „Sozialgeschichte der deutschen Literatur 1770-1900“ von Renate von Heydebrand, Dieter Pfau und Jörg Scbönert (Tübingen 1988).

${ }^{45} \mathrm{Vgl}$. den Forschungsbericht von Gerhard Sauder, Sozialgeschichtliche Aspekte der Literatur im 18. Jahrhundert, in: Internationales Archiv für Sozialgeschichte der deutschen Literatur 4 (1979) $197-241$ 
tionen verbunden: homogene und stabile vs. heterogene und mobile Sozialstruktur, konsistentes vs. inkonsistentes Normensystem, partikularistische vs. universalistische Werte, geringe vs. zahlreiche technische Innovationen, geringer vs. hoher Alphabetismus, personale vs. mediale Kommunikation, allgemeine vs. spezialisierte Rollen, einfache und stabile vs. ausdifferenzierte und wechselnde Berufe, religiös-dogmatische vs. säkularisierte Weltbilder, ländliche vs. städtische Siedlungsweise, Großfamilie vs. Kleinfamilie usw. ${ }^{46}$. Inzwischen hat die ,zweite Generation' von Modernisierungstheorien ein geschärftes Bewußtsein dafür entwickelt, daß sich in konkreten historischen Verhältnissen und Prozessen Merkmale der Traditionalität und Modernitāt nicht gegenseitig ausschließen und sogar ohne notwendige Konflikte nebeneinander bestehen können, daß Modernisierung in einem Bereich zur Traditionalisierung in einem anderen führen kann und umgekehrt. „Die Modernisierungsprozesse der einzelnen Gesellschaften stellen je eigene ,Mischungsverhältnisse (von Merkmalen der Traditionalität und Modernität, Th. A.) dar, von denen die spezifischen Konflikte und auch die Dynamik der jeweiligen Entwicklung bestimmt werden. ${ }^{\text {«7 }}$ „Statt linearem Aufstieg und totalem Sieg des Neuen“, so Hans-Ulrich Wehler in seiner einschlägigen Schrift, „kann erst das Nebeneinander von Alt und Neu, können ihre Vermischung, Kollaboration und schleichende Mutation, die Verstārkung von Modernem durch Traditionelles, von Traditionellem durch Modernes die Spannungen des realhistorischen Prozesses erfassen helfen. “48

Folgt man einer nicht eben schlecht belegten These von Alberto Martino, so ist beispielsweise die „Emanzipation“ der bürgerlichen Intelligenz von höfischen Normen bedingt durch eine im frühen 18. Jahrhundert verringerte soziale Mobilität, die es den bürgerlichen Eliten nicht mehr in dem Maße wie vorher erlaubte, sich dem Adel zu assimilieren $^{49}$. Hohe soziale Mobilität gilt als klassischer Indikator der Modernität; sie war indes, zumindest was die intellektuelle Elite angeht, im 17. weitaus höher als im 18. Jahrhundert, in dem die Literaturgesellschaft (d.i. das „System “50 der literaturbezogenen Rollen und Institutionen) ihrerseits wiederum viele Merkmale der Modernität aufzuweisen beginnt. Welche Merkmale sind das?

Modernisierungsprozesse werden u.a. als Entwicklungen zunehmender sozialer Differenzierungen beschrieben: „etwa in Berufe, Öffentlichkeit und Privatsphäre, Staat und Kirche, zentrale und lokale Macht usw. Modernisierung wird so weitgehend ge-

\footnotetext{
${ }^{46}$ Aus dem „Dichotomien-Alphabet“ in Hans-Ulrich Webler, Modernisierungstheorie und Geschichte (Göttingen 1975) 14f. und dem Merkmalskatalog in M. Rainer Lepsius, Soziologische Theoreme über die Sozialstruktur der „Moderne“ und die „Modernisierung“, in: Studien zum Beginn der modernen Welt. Hrsg. von Reinbart Koselleck (Stuttgart 1977) 16 f.; im folgenden zitiert: Webler, Modernisierungstheorie; Lepsius, Soziologische Theoreme.

${ }^{47}$ Lepsius, Soziologische Theoreme, 18.

4 Webler, Modernisierungstheorie, 41.

4 Alberto Martino, Daniel Casper von Lohenstein. Geschichte seiner Rezeption, Bd. I 1661-1800 (Tübingen 1978) 419-435.

${ }^{\text {so }}$ Ich verwende den „System“-Begriff mit all den Vorbehalten, die unter anderen Wehler, Modernisierungstheorie, $24 \mathrm{ff}$. artikuliert hat.
} 
faßt als Prozeß beständiger sozialer Differenzierung von Rollen und Institutionen: wachsender Arbeitsteilung, Spezialisierung, Ausdifferenzierung ehemals verbundener ,Funktionen', institutionelle Autonomisierung, Fragmentierung, Atomisierung und Anomie einzelner Lebensbereiche. Dabei wird dann weiter angenommen, daß mit jeder Differenzienung eine neue Integration auf höherem Niveau einhergehe, denn sonst könne ja nicht von Differenzienung eines sozialen Systems gesprochen werden, sondern man müßte von einer Teilung des Systems sprechen." ${ }^{\text {S1 }}$ In diesem Sinne führen die sich seit etwa den zwanziger Jahren des 18. Jahrhunderts abzeichnenden und nach dem Siebenjährigen Krieg durchsetzenden Veränderungen zu einer komplex und differenziert strukturierten Literaturgesellschaft, die als ,modern“ bezeichnet werden kann und sich als ein relativ autonomes System in der Gesellschaft etabliert. Diese Veränderungen sind mittlerweile vielfach beschrieben worden ${ }^{52}$ : als das rapide Anwachsen der Zahl von Autoren, Lesern (steigende Alphabetisierung), produzierten Büchern, literaturvermittelnden Medien (Zeitschriften) und Institutionen (Buchhandlungen, Bibliotheken), als Umschlag vom intensiven zum extensiven Lesen und eines entsprechend höheren und differenzierteren Lektürebedarfs, als Säkularisierung der Lesestoffe, Professionalisierung der Schriftstellerrolle, Institutionalisierung der Literaturkritik, Organisation von Lesegesellschaften, Trennung von Verlag und Sortiment, Ausdifferenzierung des klassizistischen Gattungssystems und Ausbildung neuer Texttypen (z.B. Kinder- und Jugendliteratur), als Aufspaltung von Elite- und Unterhaltungsliteratur und dergleichen mehr.

Pierre Bourdieu hatte schon 1970 mit seinem Begriff des „intellektuellen Kräftefeldes im Sinne eines Relationssystems zwischen den Trägern des Systems geistiger Produktion“53 das gleiche Phänomen vor Augen: „daß das intellektuelle Kräftefeld als autonomes oder doch auf Autonomie pochendes System aus einem Prozeß historischer Autonomisierung und einer internen Differenzierung der Gesellschaft hervorgegangen ist“54. Und er behauptete: „In dem Maße, wie das intellektuelle Feld an Autonomie gewinnt, beansprucht der Künstler immer entschiedenere Autonomie auch für

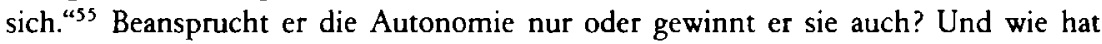
man sich diesen Zusammenhang vorzustellen?

Generell kann man wohl sagen, daß der Autor an dem Autonomiegewinn des „Systems“ der Literaturgesellschaft (bzw. des literarischen „Kräftefeldes“) partizipiert, insofern seine Abhängigkeiten von literaturexternen Ansprüchen (Theologie, Wissen-

51 Lepsius, Soziologische Theoreme, 24.

52 Siehe über die schon angegebene Forschungsliteratur hinaus unter anderem Hans Jürgen $\mathrm{Ha}$ ferkorn, Der freie Schriftsteller. Eine literar-soziologische Studie über seine Entstehung und Lage in Deutschland zwischen 1750 und 1800, in: Archiv für Geschichte des Buchwesens 5 (1964) 523-712; Wolfgang von Ungern-Sternberg, Schriftsteller und literarischer Markt, in: Hansers Sozialgeschichte der deutschen Literatur Bd. 3. Deutsche Aufklārung bis zur Französischen Revolution 1680-1789 (München 1980) 133-185; im folgenden zitiert als: Haferkorn, Der freie Schriftsteller; Ungern-Sternberg, Schriftsteller und literarischer Markt.

${ }^{53}$ Bourdieu, Soziologie der symbolischen Formen, 82.

54 Ebd. 85.

55 Ebd. 80. 
schaft, Recht, Politik, Moral usw.) sinken oder zumindest indirekter werden. Andererseits sieht sich der Autor innerhalb des literaturgesellschaftlichen Kräftefeldes neuen Anforderungen gegenüber. Die auf dem Prinzip der freien und erweiterten Konkurrenz gegründeten ,Gesetze‘ des expandierenden Buch- und Medienmarkts vergrößern den Individualitätsspielraum oder fordern sogar innovatorische Qualitäten, sie dynamisieren den Wandel und die Pluralisierung von Normen, doch unterstellen sie den Autor zugleich komplexer gewordenen Mechanismen sozialer Kontrolle.

In rollentheoretischer Perspektive läßt sich die Modernisierung der deutschsprachigen Literaturgesellschaft auch als Prozeß der Ausdifferenzierung des Bezugsgruppengeflechtes darstellen, das an den Autor normative Ansprüche stellt. Je komplexer das System einer Literaturgesellschaft (und das gilt generell für Sozialsysteme) ist, desto uneinheitlicher und pluralistischer sind die literarischen Normen in ihr. Das erweitert die Individualitätsspielräume des einzelnen Autors. Er kann einzelne Institutionen und ihre Erwartungen ohne größeren Schaden ignorieren und sich an anderen Bezugsgruppen orientieren, deren Erwartungen den eigenen Ansprüchen und Fähigkeiten am ehesten entsprechen. Autonomie in "modernen“ Literaturgesellschaften ist nicht gleichzusetzen mit der globalen Zurückweisung von Publikumserwartungen; der Konformitätsdruck von literarischen Freundeskreisen, elitären Künstlergruppen oder bestimmten Verlagen kann unter Umständen sehr stark sein. Aber der Autor hat die (begrenzte) Möglichkeit, Priorităten in der Wahl seiner Adressaten und sanktionsmächtigen Bezugsgruppen zu setzen.

Die Konfrontation mit unterschiedlichen, wenn nicht konträren Bezugsgruppen in der Literaturgesellschaft lāßt sich freilich nur begrenzt durch solche Wahlmöglichkeiten ,bewältigen', sie sind vielmehr in hohem Maße konfliktträchtig und fordern dem Autor über die situationsgemäße Konkretisierung mehr oder weniger strikt vorgegebener Normen hinaus zusätzliche Ich-Leistungen ab. Literarische Texte sind oft das Produkt schwieriger Kompromißbildungen: Der Autor muß einige kreative Anstrengungen investieren, wenn er den Erwartungen einer Bezugsgruppe (etwa den ökonomisch orientierten des Verlags) entsprechen möchte, ohne die damit nicht identischen einer anderen (z. B. des Freundeskreises) zu enttäuschen ${ }^{56}$. Integrative Leistungsanforderungen zur Bewältigung solcher Intra-Rollenkonflikte verstärken sich in dem Maße, in dem die Pluralität der den Autor gleichzeitig fordernden Bezugsgruppen zunimmt.

Eine weitere Erscheinung des literaturgesellschaftlichen Modernisierungsprozesses, die ebenfalls erweiterte Freirãume und zugleich neue, die Integrationskräfte des Ichs fordernde Konflikte schafft, ist die wachsende Zahl der Rollen, die eine Person im (und neben dem) literarischen Leben zu spielen vermag. Schon im 18. Jahrhundert trifft man auf jenen „modernen“ und universalen Typus des „freien Schriftstellers“, der die verschiedenen, im Prozeß der Ausdifferenzierung der Literaturgesellschaft heraus-

\footnotetext{
${ }^{56}$ Der literaturwissenschaftliche Begriff des „intendierten Lesers“ oder der „Leserrolle“, der/die in der Textanalyse rekonstruierbar ist, vernachlässigt diesen Kompromißbildungscharakter von Texten. Diese Begriffe müßten stets im Plural gebraucht werden.
} 
gebildeten Rollen mit ihren jeweils eigenen Anforderungen gleichzeitig oder wechselnd zu besetzen vermag, der also z. B. „das Amt des Dichters mit den Funktionen des Tagesschriftstellers, Redakteurs, Herausgebers, Kritikers, Literaturwissenschaftlers und Dramaturgen ${ }^{457}$ verbindet. Das Interagieren in so vielen verschiedenen Rollen mit jeweils anderen Normprofilen kam der Autonomie der Persönlichkeit insofern zugute, als sie die Abhängigkeit von sanktionsmächtigen Bezugsgruppen streuen konnte. Mißerfolge in der Rolle des Wissenschaftlers oder Dramaturgen können eher riskiert werden, wenn sie sich durch Erfolge in der Rolle des Kritikers oder Verlegers ausgleichen lassen. Mit der wachsenden Zahl der Rollen, die der einzelne im Leben zu spielen hat, wachsen indes auch die Belastungen durch Inter-Rollenkonflikte. Zu einem nachgerade klassischen Konflikt wird in der modernen Literaturgesellschaft der zwischen Schriftsteller- und Berufsrolle. Doch war er von etlichen Rollenkonflikten anderer Art begleitet. Eine im 18. Jahrhundert ungemein beliebte Strategie zu ihrer Bewältigung lag in anonymen oder pseudonymen Veröffentlichungen. Wenn die Erwartungen an einen Verfasser, galanter Romane nicht in Einklang zu bringen waren mit den Erwartungen an den Literaturkritiker oder Tragödiendichter oder auch mit der außerliterarischen Rolle des Beamten, dann bot das Verschweigen des eigenen Namens eine relativ leichte Lösung des Konfliks ${ }^{58}$.

Gerade diese Art der Konfliktlösung verweist besonders anschaulich auf die spezifische Schwierigkeit, die dem in modernen, komplexen Gesellschaften geforderten Spiel in vielen Rollen (nacheinander, nebeneinander oder zugleich) innewohnt. Sie liegt für das Ich darin, gegenüber all den Differenzen in dem, was ihm in einem ausdifferenzierten Rollen- und Normgefüge abverlangt wird, eine sie ausbalancierende Identität zu bewahren, sich als „Individuum“, als unteilbar zu behaupten. Ich erinnere in diesem Zusammenhang noch einmal an das Modernisierungstheorem, demzufolge mit jeder Differenzierung (die in ihren negativen Aspekten auch als Fragmentierung oder Atomisierung und in ihren positiven als leistungssteigernde Komplexitätserweiterung beschrieben wird) eine neue Integration auf höherem Niveau einhergeht, weil sonst nicht von Differenzierung, sondern von Teilung eines sozialen Systems gesprochen werden mußs. In dieser Perspektive beschwört das für moderne Gesellschaften charakteristische Individualitātspathos, für das die Genieāsthetik des 18. Jahrhunderts nur ein ausgeprāgtes Beispiel liefert, nicht zuletzt den hohen und abstrakten Wert einer integrativen Kraft oder, um vom systemtheoretischen Vokabular in das des 18. Jahrhunderts überzuwechseln, eines ganzheitlichen Vermögens, das, darin den emphatischen Begriffen des Ästhetischen, der Humanitāt oder der Bildung ãquivalent, der stets drohenden Atomisierung in isolierte Einzelteile entgegensteht. Die individuelle Ich-Identităt entspricht mithin als ein sozialer Wert den Anforderungen komplexer Sozialsysteme an die Persönlichkeit, doch zugleich trägt sie in sich ein widerständiges Potential. Denn nur indem sie sich der Anpassung an partikulare Gruppeninteressen verweigert, sich von ihnen nicht, auseinanderdividieren' läßt, sondern sich ihnen ge-

s7 Haferkorn, Der freie Schriftsteller, 526.

${ }^{58} \mathrm{Vgl}$. Ungern-Sternberg, Schriftsteller und literarischer Markt, 170-173. 